\title{
Prevalencia del hígado graso no alcohólico y su asociación con alteraciones bioquímicas en una población mexicana asintomática
}

\author{
Prevalence of non-alcoholic fatty liver and its association with \\ biochemical alterations in asymptomatic Mexican population
}

\author{
María José Ortega Chavarría, ${ }^{*}$ Georgina Cornelio Rodríguez, ${ }^{\ddagger}$ \\ Federico Rodríguez Weber, ${ }^{\S}$ Enrique Díaz Greene ${ }^{\S}$
}

\section{Resumen}

El hígado graso no alcohólico (HGNA) se ha convertido en la causa más frecuente de enfermedad hepática crónica a nivel mundial y se considera como una manifestación hepática del síndrome metabólico. La mayoría de los estudios epidemiológicos proceden de técnicas no invasivas, como el ultrasonido. Objetivo: Evaluar la presencia de HGNA por ultrasonido y su asociación con alteraciones bioquímicas relacionadas con el síndrome metabólico sin tomar en cuenta medidas antropométricas. Material y métodos: Estudio observacional, transversal y retrospectivo, el cual se realizó en aquella población mexicana que acudió a la Clínica de Diagnóstico del Hospital Ángeles Pedregal, en Ciudad de México, entre el 1ero. de mayo al 31 de agosto de 2018, con un total de 513 casos. Resultados: La prevalencia encontrada de HGNA por ultrasonido fue del $28.65 \%$ (147 casos). Se demostró que las cifras séricas de glucosa, triglicéridos, colesterol HDL y VLDL, aspartato y alanina-aminotransferasa contaban con una p estadísticamente significativa; de la misma manera, la asociación con el género masculino y el incremento de la edad también se encontraron asociadas con el HGNA. Conclusiones: La presencia de HGNA por ultrasonido, encontrada aun de forma incidental, debería impulsar a que los clínicos iniciaran un abordaje metabólico completo.

Palabras clave: Hígado graso no alcohólico, variables bioquímicas, síndrome metabólico, ultrasonido de abdomen.

\section{Abstract}

Non-alcoholic fatty liver disease (NAFLD) has become the most frequent cause of chronic liver disease worldwide, is considered the hepatic manifestation of metabolic syndrome. Most epidemiologic studies derive from non-invasive techniques, such as the ultrasound. Objective: To evaluate the presence of NAFLD by ultrasound and its association with biochemical alterations with metabolic syndrome, without taking into consideration anthropometric measures. Material and methods: Observational, transversal and retrospective study. It was performed in asymptomatic and apparently healthy Mexican population that had a check up performed at the Diagnostic Clinic at Hospital Ángeles Pedregal, Mexico City in the time frame between 1st of May to the 31st of August 2018 with a total of 513 cases. Results: Prevalence of NAFLD found by ultrasound was $28.65 \%$ (147 cases). It was shown that serum glucose, triglyceride, HDL and VLDL cholesterol levels, aspartate and alanine aminotransferase had statistically significant $p$; the association with the male gender and the increase in age were also associated with HGNA. Conclusion: The presence of NAFLD by ultrasound, even incidentally, should compel clinicians to start a complete metabolic diagnosis.

Keywords: Non-alcoholic fatty liver disease, biochemical variables, metabolic syndrome, abdominal ultrasound.
* Residente del curso de Medicina Interna. Facultad Mexicana de Medicina de la Universidad La Salle. Ciudad de México.

₹ Residente del curso de Imagenología, Diagnóstico y Terapéutica. Facultad de Medicina de la Universidad Autónoma de México. Ciudad de México.

$\S$ Profesor del curso de Medicina Interna. Facultad Mexicana de Medicina de la Universidad La Salle. Ciudad de México.

www.medigraphic.com/actamedica
Hospital Ángeles Pedregal. Ciudad de México, México.

Correspondencia:

Dra. María José Ortega Chavarría

Correo electrónico: dra.mariajose.ortega@gmail.com

Aceptado: 21-08-2019.

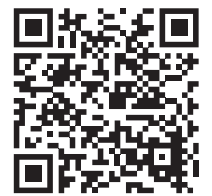




\section{INTRODUCCIÓN}

El hígado graso no alcohólico (HGNA) es la enfermedad hepática más común a nivel mundial ${ }^{1}$ y se ha convertido en la primera causa de trasplante hepático en México. Éste es el resultado de una compleja interacción entre factores ambientales y genéticos. Su incidencia ocurre en todos los grupos etarios en población mexicana, especialmente en aquellas personas entre los 40 y 59 años, aunque también se ha relacionado con el sexo masculino y los polimorfismos PNPLA3. ${ }^{2}$

Esta patología se define por la acumulación de triglicéridos a nivel citoplasmático en más del 5\% de los hepatocitos en ausencia de un consumo significativo de alcohol ( $<20 \mathrm{~g} /$ día), uso de medicamentos hepatotóxicos, alguna infección viral u otra patología hepática preexistente. ${ }^{3} \mathrm{La}$ prevalencia reportada en la población general asintomática en México es del $17.1 \%{ }^{4} \mathrm{y}$, a nivel mundial, va desde el 10 al 24\%, con un incremento hasta de 4.6 veces más en la población obesa. ${ }^{5}$ Otros factores asociados son: la presencia de hiperinsulinemia, la dislipidemia, las alteraciones metabólicas de la glucosa, la diabetes mellitus tipo 2 y la circunferencia abdominal (mayor a $102 \mathrm{~cm}$ en hombres y a $88 \mathrm{~cm}$ en mujeres).

El HGNA es considerado como una manifestación hepática del síndrome metabólico, siendo un órgano activo en la patogénesis y sus complicaciones posteriores. ${ }^{6}$ El término HGNA hace referencia al espectro de daño hepático, que va desde la simple esteatosis (estado inflamatorio) hasta el daño celular con la balonización de los hepatocitos y el desarrollo de fibrosis (esteatohepatitis). Esta manifestación hepática puede progresar hacia una cirrosis en el $22 \%$ de los casos dentro de un periodo de 10 años; en este subgrupo, hasta un $27 \%$ desarrollará un carcinoma hepatocelular. ${ }^{7,8}$

El diagnóstico de HGNA se realiza de forma incidental, ya que la mayoría de los pacientes suelen encontrarse asintomáticos y, hasta en un $50 \%$ de los casos, sus niveles de transaminasas se hallan en rangos normales. ${ }^{9}$ Estudios recientes han demostrado que el aumento de la beta oxidación de ácidos grasos, el estrés oxidativo y la resistencia periférica a la insulina están presentes en todos los sujetos con hígado graso no alcohólico, aun en ausencia de diabetes mellitus tipo $2 .^{10}$ El estándar de oro para el diagnóstico es la toma de biopsia hepática, aunque en la mayoría de los casos no existe alguna indicación médica y ésta no tiene relevancia para la toma de decisiones terapéuticas. ${ }^{11}$ Otro estudio de utilidad, de bajo costo y de poca invasividad es el ultrasonido de abdomen, el cual puede identificar la infiltración grasa hepática; cuando hay presencia de depósitos difusos o locales, se suele clasificar en leve, moderada o severa. A continuación se describen cada una de ellas:

Leve. Existe un aumento difuso y mínimo de la ecogenicidad hepática, además de una adecuada visualización del diafragma y el borde de los vasos intrahepáticos.

Moderada. Hay un aumento difuso moderado de la ecogenicidad hepática, así como una ligera pérdida de la visualización de los vasos intrahepáticos y el diafragma.

Severa. Se observa un aumento marcado de la ecogenicidad hepática con una mala penetración del segmento posterior del lóbulo derecho hepático y mala o nula visualización de los vasos hepáticos y del diafragma. ${ }^{12}$ Hay que tener en cuenta que la sensibilidad de este estudio es limitada cuando el grado de infiltración grasa es menor al $30 \%$ y la interpretación es operador-dependiente.

Otras herramientas de utilidad para el diagnóstico son dos: la primera, es la elastografía (FibroScan ${ }^{\circledR}$ ), que mide la rigidez hepática y correlaciona con el grado de fibrosis y, la segunda, la resonancia magnética, que identifica con exactitud cercana al $100 \%$ la presencia de esteatosis en porcentajes mayores al $5.56 \%$. El uso de ambas técnicas se encuentra actualmente limitada debido a su poca disponibilidad en centros de atención, además de que su costo es elevado. ${ }^{13-15}$

Otro dato importante a comentar es la existencia de dos diferentes fenotipos. En uno, existen individuos en peso e índice de masa corporal (IMC) normal, pero con datos de pacientes metabólicamente obesos, los cuales presentan datos de resistencia a la insulina, adiposidad central, cifras bajas de colesterol de baja densidad (HDL) y elevadas concentraciones de triglicéridos, pudiendo estar o no relacionadas con hipertensión arterial sistémica; en el otro, existen obesos metabólicamente sanos, los cuales no presentan ninguna de las alteraciones metabólicas mencionadas, pero sí un IMC > $30 .{ }^{16}$ Se ha demostrado que la acumulación local de grasa en depósitos viscerales es más importante que la cantidad de grasa corporal total, lo que constituye un factor predictor más confiable de riesgo cardiovascular. ${ }^{17,18}$ La disfunción del tejido adiposo y la resistencia a la insulina aparecerían una vez que los mecanismos de respuesta compensadora se encuentren agotados. ${ }^{19}$

Objetivo.Evaluar la presencia del hígado graso no alcohólico por medio del ultrasonido y su asociación con alteraciones bioquímicas que se relacionan con el síndrome metabólico, sin tomar en cuenta medidas antropométricas.

\section{MATERIAL Y MÉTODOS}

Estudio observacional, transversal y retrospectivo. Se llevó a cabo en una población mexicana asintomática y aparente- 
mente sana, la cual acudió a chequeo médico en la Clínica de Diagnóstico del Hospital Ángeles Pedregal, en la Ciudad de México, durante el periodo comprendido del 1ero. de mayo al 31 de agosto de 2018. En este estudio se tuvo un total de 522 pacientes, de los que se descartaron nueve casos por no contar con los criterios de inclusión completos, teniendo, por tanto, 513 casos como la población final.

Criterios de inclusión: se tomaron en cuenta a todos los pacientes mexicanos mayores de 18 años, quienes hubieran acudido a valoración en la Clínica de Diagnóstico del Hospital Ángeles Pedregal durante el periodo de tiempo establecido y que contaran con laboratorios y ecografía abdominal en la misma valoración.

Criterios de exclusión: se eliminaron del estudio a pacientes menores de 18 años, que fueran extranjeros, embarazadas o que tuvieran alguna enfermedad hepática conocida o consumo de alcohol $>20$ g/día (hombres) y $>10$ g/día (mujeres), uso crónico de medicamentos conocidos como hepatotóxicos, antecedente de enfermedades reumatológicas y aquéllos con laboratorios o gabinete incompletos.

Variables bioquímicas: todas las tomas de muestras se realizaron acorde con los protocolos establecidos por la institución, de tal manera que se les pidió a todos los pacientes que tuvieran un tiempo de ayuno mínimo de ocho horas. Posteriormente, se analizaron y clasificaron los pacientes por género y edad; se tomaron en cuenta los criterios de ATP III (panel de tratamiento para del adulto del Programa Nacional de Educación sobre el Colesterol) para definir la alteración de glucosa de ayuno con cifras $\geq$ $110 \mathrm{mg} / \mathrm{dL}$, colesterol HDL $\leq 40 \mathrm{mg} / \mathrm{dL}$ en hombres $y \leq 50$ $\mathrm{mg} / \mathrm{dL}$ en mujeres, y triglicéridos $\geq 150 \mathrm{mg} / \mathrm{dL}$. Además, se midieron otras variables, como colesterol LDL > $160 \mathrm{mg} /$ $\mathrm{dL}$, colesterol $\geq 200 \mathrm{mg} / \mathrm{dL}$, albúmina $<3.5 \mathrm{~g} / \mathrm{dL}$, alaninaaminotransferasa (TGP) $>66 \mathrm{U} / \mathrm{L}$ y aspartato aminotransferasa (TGO) $>55 \mathrm{U} / \mathrm{L}$, que se conocen como parámetros asociados. Los exámenes de laboratorio fueron medidos con la técnica de rutina mediante colorimetría y enzimática.

Variables por estudio de imagen: se realizaron rastreos ecográficos abdominales con protocolo para abdomen superior o abdomen completo, con equipos Philips ${ }^{\circledR}$ modelos iU22 y EPIQ G7 utilizando transductores convexos (Philips ${ }^{\circledR}$ C5-1) con frecuencia de 5-1 MHz y apertura de banda de $55.5 \mathrm{~mm}$. Las imágenes obtenidas fueron enviadas al sistema CARESTREAM Vue Motion ${ }^{\circledR}$. Tomando en cuenta la ecogenicidad del parénquima hepático, se clasificaron en hígado sano e hígado graso; en este último, a su vez, se subclasificó en leve, moderado y severo de acuerdo con los criterios ecográficos establecidos y comentados previamente. ${ }^{20}$ Los estudios fueron interpretados posterior a su realización y al momento de la recolección de datos, sin encontrar diferencias entre ambas interpretaciones.

\section{Análisis estadístico}

Las variables fueron ordenadas en una base de datos en el programa Excel ${ }^{\circledR}$ de Microsoft ${ }^{\circledR}$ bajo absoluta confidencialidad para su posterior análisis con el paquete estadístico RStudio ${ }^{\circledR}$. Para todas las pruebas, se adoptó un nivel de significancia alfa de 0.05 .

De cada muestra, se realizó un análisis univariado y descriptivo, donde para las variables cuantitativas se calcularon medias, desviación estándar (DE) y se comprobó el ajuste a una distribución normal con la prueba Shapiro-Wilk. Para variables dicotómicas, se calcularon las frecuencias observadas y su correspondiente validación general y por grupo. Se continuó con un análisis bivariado, comparando variables dicotómicas y realizando tablas de contingencia de 2 por 2; para contrastar la hipótesis de independencia entre variables, se utilizó la prueba $\chi^{2}$ de Pearson. Para variables cuantitativas, se hizo el contraste con ayuda de la prueba no paramétrica $U$ de Mann Whitney. Se reportó su correspondiente valor de significancia por valor de $p$.

Finalmente, se realizó un modelo de regresión logística para predecir la presencia de hígado graso no alcohólico, y se utilizó la prueba de Hosmer y Lemeshow para evaluar la bondad de ajuste del modelo.

\section{RESULTADOS}

Se tomaron en cuenta 513 casos, cuya edad promedio fue de 47.35 años ( $D E=11.24)$. De éstos, 276 eran mujeres (53.8\%), y 237 hombres (46.2\%) (Figura 1).

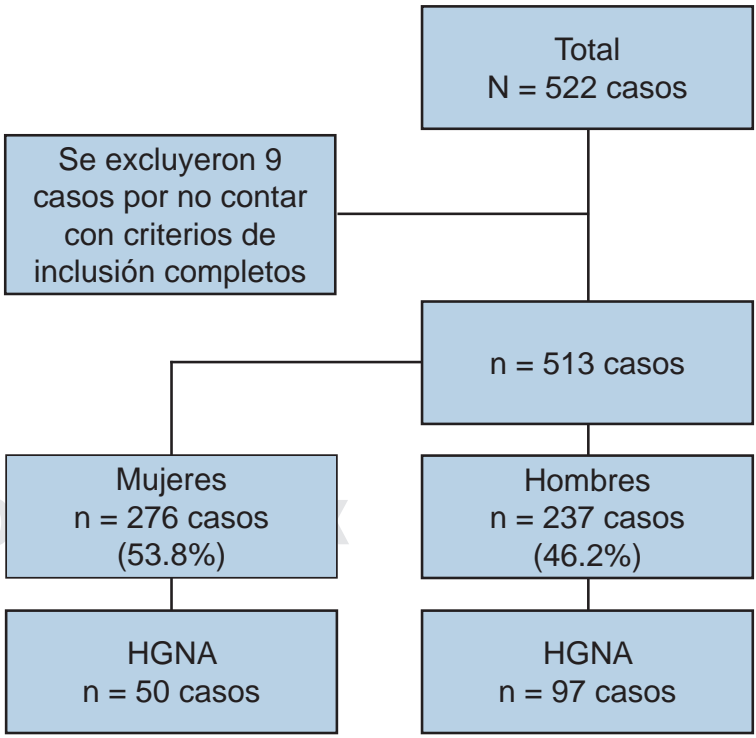

Figura 1: Distribución de la población estudiada. HGNA = hígado graso no alcohólico. 
Tabla 1: Características demográficas de la población estudiada ( $\mathrm{N}=513$ casos).

\begin{tabular}{|c|c|c|c|c|}
\hline & \multicolumn{2}{|c|}{ Mujeres } & \multicolumn{2}{|c|}{ Hombres } \\
\hline Población, n (\%) & \multicolumn{2}{|c|}{$276(53.8)$} & \multicolumn{2}{|c|}{$237(46.2)$} \\
\hline Edad (años), media \pm DE & \multicolumn{2}{|c|}{$46.07 \pm 11.3$} & \multicolumn{2}{|c|}{$48.84 \pm 11$} \\
\hline Casos (n) & $\begin{array}{l}\text { Sí } \\
50\end{array}$ & $\begin{array}{l}\text { No } \\
226\end{array}$ & $\begin{array}{l}\text { Sí } \\
97\end{array}$ & $\begin{array}{l}\text { No } \\
140\end{array}$ \\
\hline \multicolumn{5}{|c|}{ Variables bioquímicas, media $\pm \mathrm{DE}$} \\
\hline Lípidos totales (mg/dL) & $650.98 \pm 196.62$ & $588.80 \pm 121.54$ & $692.01 \pm 253.59$ & $574.28 \pm 148.28$ \\
\hline Triglicéridos (mg/dL) & $169.1 \pm 85.89$ & $113.47 \pm 50.36$ & $188.52 \pm 115.62$ & $126.77 \pm 60.59$ \\
\hline Colesterol (mg/dL) & $192.6 \pm 40$ & $196.95 \pm 34.73$ & $196.03 \pm 43.32$ & $194.48 \pm 35.60$ \\
\hline $\mathrm{HDL}(\mathrm{mg} / \mathrm{dL})$ & $45.90 \pm 12.88$ & $56.53 \pm 14.08$ & $40.76 \pm 9.04$ & $46.71 \pm 12.52$ \\
\hline $\mathrm{LDL}(\mathrm{mg} / \mathrm{dL})$ & $115.32 \pm 33.27$ & $117.69 \pm 31.11$ & $119.18 \pm 35.92$ & $123.18 \pm 31.31$ \\
\hline VLDL (mg/dL) & $33.96 \pm 22.65$ & $23.44 \pm 13.12$ & $36.24 \pm 21.44$ & $25.05 \pm 11.35$ \\
\hline Glucosa (mg/dL) & $101.5 \pm 33.79$ & $87.52 \pm 16.05$ & $96.35 \pm 15.61$ & $93.21 \pm 48.44$ \\
\hline TGP (U/L) & $36 \pm 21.56$ & $23.02 \pm 13.25$ & $40.81 \pm 27.75$ & $29.86 \pm 14.05$ \\
\hline TGO (U/L) & $27.26 \pm 12.62$ & $21.36 \pm 7.37$ & $28.29 \pm 14.80$ & $24.81 \pm 9.60$ \\
\hline Albumina (g/dL) & $4.04 \pm 0.24$ & $4.01 \pm 0.28$ & $4.19 \pm 0.26$ & $4.20 \pm 0.32$ \\
\hline
\end{tabular}

Abreviaturas (por sus siglas en inglés): $\mathrm{DE}=$ desviación estándar, $\mathrm{HDL}=$ lipoproteínas de alta densidad, LDL = lipoproteínas de baja densidad, VLDL = lipoproteína de muy baja densidad, TGP = alanina-aminotransferasa, $T G O=$ aspartato aminotransferasa.

De la población total, se evidenció la presencia de hígado graso no alcohólico (HGNA) por ultrasonido en 147/513 casos (28.65\%). De esta proporción, el patrón ecocardiográfico más prevalente fue el grado moderado en 74/147 casos (50.34\%). En la Tabla 1, se muestran las características demográficas de la población total, marcando el promedio y desviación estándar de las variables bioquímicas, así como la relación con la presencia o ausencia de HGNA por ultrasonido.

La Tabla 2 presenta la distribución de la población que contaba con hígado graso no alcohólico (HGNA) e hígado normal y su relación con las variables bioquímicas. Se puede observar que las variables de laboratorio (lípidos totales, triglicéridos, colesterol VLDL, colesterol HDL, glucosa, TGO y TGP) resultaron estar relacionadas con un HGNA de forma estadísticamente significativa.

Para estudiar el comportamiento entre variables estadísticamente significativas asociadas con la presencia de HGNA, se realizó un modelo de regresión logística (Tabla 3), ajustado por lípidos totales, triglicéridos, VLDL, HDL, glucosa y enzimas hepáticas y controlado por edad y género (variable dicotómica donde 1: mujer, y 0: hombre). Por último, las variables demográficas de sexo y edad resultaron ser estadísticamente significativas al compararlas con nuestra variable respuesta $(p<$ $0.0001 \operatorname{con} \chi^{2}=31.35 \%$ y $p=0.028$ de una prueba $U$ de Mann-Whitney, respectivamente).

\section{DISCUSIÓN}

A pesar de que la mayoría de los estudios hechos hasta el momento toman en cuenta valores antropométricos (peso, talla, circunferencia abdominal e índice de masa corporal), en el presente estudio, no se tomaron en cuenta, debido al interés por conocer la relevancia del depósito de lípidos a nivel hepático por ultrasonido y su asociación con alteraciones bioquímicas de forma independiente a estos parámetros. Los resultados obtenidos fueron de relevancia, pues al respecto, se encontró más prevalencia que, por ejemplo, la reportada previamente por López-Velázquez JA y colaboradores, ${ }^{4}$ pues en nuestro caso se reportaron $147 / 513$ casos (28.65\%) de la población estudiada, siendo el grado moderado el patrón que más frecuente se reportó en 74 de los 147 casos (50.34\%), lo cual es más cercano a la prevalencia mundial reportada.

Al analizar las variables bioquímicas, se encontró que éstas se relacionaron con la presencia de HGNA, como era de esperarse, siendo las mismas que se toman en cuenta para el diagnóstico de síndrome metabólico según los criterios del ATP III: glucosa, triglicéridos y colesterol HDL, existiendo también un involucramiento de los niveles de colesterol de muy baja densidad (VLDL, por sus siglas en inglés), aspartato y alanina-aminotransferasa (TGO y TGP, respectivamente), cuyas $p$ fueron estadísticamente significativas. Las cifras de albumina sérica no fueron 
representativas (Tabla 2). Al analizar el comportamiento en conjunto de las variables estadísticamente significativas como resultado de la Tabla 2, se encontró que los niveles de lípidos totales, triglicéridos y TGP continuaron siendo significativas para explicar la presencia de HGNA, estando en asociación con la edad y al género. Por lo que se concluye que, a mayor edad, género masculino, cifras elevadas de lípidos totales, triglicéridos y TGP, se incrementa el riesgo de presentar HGNA; en tanto ser mujer, cifras normales de glucosa sérica y cifras elevadas de HDL-colesterol reducen el riesgo (Tabla 3).

La elevación de la TGP se ha asociado con la resistencia a la insulina en tejido adiposo y con el contenido de triglicéridos a nivel hepático, sin existir buena relación con la inflamación o fibrosis. Hasta en $60 \%$ de los pacientes con HGNA confirmado mediante biopsia presentan cifras normales de esta enzima hepática, de modo que es un mal indicador para el diagnóstico y seguimiento de forma

Tabla 2: Distribución de hígado graso no alcohólico e hígado sano respecto a variables bioquímicas.

\begin{tabular}{|c|c|c|c|}
\hline & \multicolumn{2}{|c|}{ Hígado graso no alcohólico } & \multirow[b]{2}{*}{$p^{*}$} \\
\hline & $\begin{array}{l}\text { Sí }(n=147) \\
\text { Media } \pm \text { DE }\end{array}$ & $\begin{array}{c}\text { No }(n=366) \\
\text { Media } \pm \text { DE }\end{array}$ & \\
\hline Lípidos totales & $678.05 \pm 235.98$ & $564.72 \pm 132.43$ & $<0.0001^{\ddagger}$ \\
\hline Colesterol & $194.86 \pm 42.11$ & $196.01 \pm 35.04$ & 0.955 \\
\hline Triglicéridos & $181.91 \pm 106.54$ & $118.56 \pm 54.80$ & $<0.0001^{\ddagger}$ \\
\hline LDL & $117.86 \pm 34.97$ & $119.76 \pm 31.26$ & 0.950 \\
\hline VLDL & $35.46 \pm 21.81$ & $24.06 \pm 12.48$ & $<0.0001^{\ddagger}$ \\
\hline HDL & $42.52 \pm 10.76$ & $52.77 \pm 14.31$ & $<0.0001^{\ddagger}$ \\
\hline Glucosa & $98.10 \pm 23.44$ & $89.70 \pm 32.56$ & $<0.0001^{\ddagger}$ \\
\hline TGP & $39.17 \pm 25.84$ & $25.63 \pm 13.94$ & $<0.0001^{\ddagger}$ \\
\hline TGO & $27.94 \pm 14.06$ & $22.68 \pm 8.45$ & $<0.0001^{\ddagger}$ \\
\hline Albumina & $4.14 \pm 0.26$ & $4.08 \pm 0.31$ & 0.016 \\
\hline
\end{tabular}

* Prueba U de Mann-Whitney. ${ }^{\ddagger}$ Significancia estadística.

Abreviaturas (por sus siglas en inglés): DE = desviación estándar, LDL = lipoproteínas de baja densidad, VLDL = lipoproteína de muy baja densidad, HDL = lipoproteínas de alta densidad, TGP = alanina-aminotransferasa, TGO = aspartato aminotransferasa.

\begin{tabular}{lcccc}
\multicolumn{2}{c}{ Tabla 3: Modelo de regresión logística. } & & \\
\hline Variables incluidas en el modelo & Razón de momios & Error estándar & Valor z & Pr(>|z|) \\
\hline Intercepto & & & & \\
Género & 0.068 & 0.875 & -3.078 & 0.002 \\
Edad & 0.545 & 0.237 & -2.564 & 0.010 \\
Lípidos totales & 1.027 & 0.010 & 2.545 & 0.011 \\
Triglicéridos & 0.996 & 0.002 & -2.141 & 0.032 \\
VLDL & 1.020 & 0.006 & 3.120 & 0.002 \\
HDL & 0.992 & 0.024 & -0.349 & 0.727 \\
Glucosa & 0.989 & 0.008 & -1.365 & 0.172 \\
TGP & 0.999 & 0.004 & -0.142 & 0.887 \\
TGO & 1.035 & 0.009 & -0.183 & $<$ \\
\hline
\end{tabular}

Prueba Hosmer-Lemeshow para probar bondad de ajuste del modelo: valor $p=0.7138$ y $\chi^{2}=5.421$

Abreviaturas (por sus siglas en inglés): VLDL = lipoproteína de muy baja densidad, $\mathrm{HDL}=$ lipoproteínas de alta densidad, TGP = alanina-aminotransferasa, TGO = aspartato aminotransferasa. 
aislada. ${ }^{21}$ En este sentido, el uso de ecografía como marcador de almacén de lípidos a nivel visceral fue útil para lograr un acercamiento al diagnóstico de hígado graso no alcohólico, siendo indicativo de anormalidad metabólica corroborado por los parámetros bioquímicos previamente mencionados, aun sin evidencia de sobrepeso u obesidad.

El estudio ecográfico es un método rápido, eficaz y de bajo costo para el tamizaje de población general, con una sensibilidad y especificidad razonable en la detección de HGNA, principalmente en grados moderados y severos, siempre tomando en cuenta que es un estudio operador dependiente. Saadeh y su quipo de investigación demostraron a través de un estudio prospectivo que, al comparar el ultrasonido en comparación con la biopsia hepática, la identificación del hígado graso por el primer método tuvo una sensibilidad del $94 \%$ y una especificidad del $84 \%{ }^{22}$ Por ello, la estrecha asociación entre la presencia de HGNA y las variables bioquímicas relacionadas con alteraciones metabólicas debe hacer hincapié en la prevención de síndrome metabólico para el desarrollo de esta patología.

\section{CONCLUSIONES}

En este contexto, la presencia de hígado graso no alcohólico (HGNA) por ultrasonido, encontrado aun de forma incidental, debería impulsara a que los clínicos iniciaran un abordaje metabólico completo, en el cual se incluya la toma de perfil lipídico, niveles de glucosa y enzimas hepáticas, con el fin de modificar resultados a largo plazo.

En el presente estudio, nuestra población se comportó de forma similar a la reportada en la literatura mundial, cuya prevalencia fue de $28.65 \%$ de HGNA del total de casos estudiados. La asociación con cifras elevadas de lípidos totales, triglicéridos, aspartato transaminasa, así como la edad y el sexo masculino fueron estadísticamente significativas incluso sin evidencia de sobrepeso u obesidad. No obstante, teniendo en cuenta la amplia población sobre la que se tendría que realizar el tamizaje, son necesarios más datos para confirmar si esta estrategia es sostenible y costo-efectiva.

\section{AGRADECIMIENTOS}

Se agradece de manera particular por sus contribuciones a la realización del artículo a la Licenciada en Actuaria, Alejandra Fragoso González, y a la Dra. Erika Faride Rodríguez Aguilar.

\section{Conflictos de interés y financiamiento: Ninguno.}

\section{REFERENCIAS}

1. Kwak MS, Kim D. Non-alcoholic fatty liver disease and lifestyle modifications, focusing on physical activity. Korean J Intern Med. 2018; 33 (1): 64-74.
2. Bernal-Reyes R, Castro-Narro G, Malé-Velázquez R, CarmonaSánchez R, González-Huezo MS, García-Juárez l et al. The Mexican consensus on nonalcoholic fatty liver disease. Rev Gastroenterol Mex. 2019; 84 (1): 69-99.

3. Boyce CJ, Pickhardt PJ, Kim DH, Taylor AJ, Winter TC, Bruce RJ et al. Hepatic steatosis (fatty liver disease) in asymptomatic adults identified by unenhanced low-dose CT. AJR Am J Roentgenol. 2010; 194 (3): 623-628.

4. López-Velázquez JA, Silva-Vidal KV, Ponciano-Rodríguez G, ChávezTapia NC, Arrese M, Uribe M et al. The prevalence of nonalcoholic fatty liver disease in the Americas. Ann Hepatol. 2014; 13 (2): 166-178.

5. Dixon JB, Bhathal PS, O'Brien PE. Nonalcoholic fatty liver disease: predictors of nonalcoholic teatohepatitis and liver fibrosis in the severely obese. Gastroenterology. 2001; 121: 91-100.

6. Targher G, Day CP, Bonora E. Risk of cardiovascular disease in patients with nonalcoholic fatty liver disease. N Engl Med. 2010; 363 (4): 1341-1350.

7. Wang RT, Koretz RL, Yee HF Jr. Is weight reduction an effective therapy for nonalcoholic fatty liver? A systematic review. Am J Med. 2003; 115: 554-559.

8. Wanless IR, Lentz JS. Fatty liver hepatitis (steatohepatitis) and obesity: an autopsy study with analysis of risk factors. Hepatology. 1990; 12: 1106-1110.

9. Vernon G, Baranova A, Younossi ZM. Systematic review: the epidemiology and natural history of non-alcoholic fatty liver disease and non-alcoholic steatohepatitis in adults. Aliment Pharmacol Ther. 2011; 34: 274-285.

10. Lizardi-Cervera J, Becerra LI, Chávez-Tapia NC et al. Prevalencia de hígado graso no alcohólico y síndrome metabólico en población asintomática. Rev Gastroenterol Mex. 2006; 71 (4): 453-459.

11. Goldstein BJ, Scalia R. Adiponectin: a novel adipokine linking adipocytes and vascular function. J Clin Endocrinol Metab. 2004; 89: 2563-2568.

12. Csendes GP, Paolinelli GP, Busel MD, Venturelli AV, Rodríguez J. Hígado graso: ultrasonido y correlación anatomopatológica. Rev Chil Radiol. 2004; 10 (2): 50-52.

13. Kramer H, Pickhardt PJ, Kliewer MA, Hernando D, Chen GH, Zagzebski JA et al. Accuracy of liver fat quantification with advanced $\mathrm{CT}, \mathrm{MRI}$, and ultrasound techniques: prospective comparison with MR spectroscopy. AJR Am J Roentgenol. 2017; 208 (1): 92-100.

14. Lee SS, Park SH. Radiologic evaluation of nonalcoholic fatty liver disease. World J Gastroenterol. 2014; 20: 7392-7402.

15. Reeder SB, Cruite I, Hamilton G et al. Quantitative assessment of liver fat with magnetic resonance imaging and spectroscopy. J Magn Reson Imaging. 2011; 34 (4): 729-749.

16. Allison DB, Heshka S. Toward an empirically derived typology of obese persons. Int J Obes. 1991; 15: 741-754.

17. Yusuf S, Hawken S, Ounpuu S, Bautista L, Franzosi MG, Commerford $\mathrm{P}$ et al; INTERHEART Study Investigators. Obesity and the risk of myocardial infarction in 27,000 participants from 52 countries: a case-control study. Lancet. 2005; 366: 1640-1649.

18. Yu SJ, Kim W, Kim D, Yoon JH, Lee K, Kim JH et al. Visceral obesity predicts significant fibrosis in patients with nonalcoholic fatty liver disease. Medicine (Baltimore). 2015; 94 (48): e2159.

19. Laclaustra M, Corella D, Ordovas JM. Metabolic syndrome pathophysiology: the role of adipose tissue. Nutr Metab Cardiovasc Dis. 2007; 17: 129-139.

20. Rumack C, Wilson S, Charboneau W. Diagnostic ultrasound. 2a. edición. St. Louis, MO: Mosby; 1998. pp. 110-112.

21. Maximos M, Brill F, Portillo Sanchez P et al. The role of liver fat and insulin resistance as determinants of plasma aminotransferase elevation in nonalcoholic fatty liver disease. Hepatology. 2015; 61 (1): 153-160.

22. Saadeh S, Younossi ZM, Remer EM, Gramlich T, Ong JP, Hurley M et al. The utility of radiological imaging in nonalcoholic fatty liver disease. Gastroenterology. 2002; 123: 745-750. 